\title{
Risk Factors for Infection by Extended-Spectrum Beta-Lactamase Producing Klebsiella pneumoniae in a Tertiary Hospital in Salvador, Brazil
}

\author{
Nanci Silva ${ }^{1,2}$, Márcio Oliveira², \\ Antonio Carlos Bandeira ${ }^{1}$ and Carlos Brites ${ }^{2,3}$
}

\author{
${ }^{1}$ Medicine and Public Health School of Bahia, ${ }^{2}$ Espanhol Hospital, \\ ${ }^{3}$ Federal University of Bahia; Salvador, BA, Brazil
}

\begin{abstract}
Nosocomial infection caused by extended-spectrum beta-lactamase producing Klebsiella pneumoniae (ESBL-Kp) have been frequently reported worldwide. We have no information on such problems in Bahia, Brazil. Objectives. Evaluate the risk factors for nosocomial infections caused by ESBL-Kp, in a tertiary hospital, in Bahia, Brazil. Material and Methods. We evaluated all reported cases of nosocomial infections caused by ESBL-Kp in a private, tertiary hospital, in Salvador, Brazil, from 2000 through 2004. We compared patients with a diagnosis of ESBL-Kp (cases) and patients infected by non-ESBL producing $K$. pneumoniae (controls). Mean age, underlying disease, and frequency of invasive procedures were compared between the two groups. History of previous use of antibiotics was also analyzed. Results. Based on multivariate analysis, previous use of antibiotics, diagnosis of malignant diseases, and diabetes mellitus were independent risk factors for acquisition of ESBL-Kp infection. No correlation was found for age, use of corticosteroids, diagnosis of chronic renal failure or AIDS, and infection by ESBL-Kp. Conclusion. Our findings suggest that the use of antibiotics or underlying disease that increases the chance of antibiotic are the main risk factors for ESBL-Kp infections. Programs focusing on rational use of antibiotics are mandatory for prevention and control of such infections.
\end{abstract}

Key Words: Nosocomial infections, ESBL, Klebsiella pneumoniae, bacterial resistance.

Gram-negative bacilli (GNB) are common agents of nosocomial infections. They cause infections of the respiratory (RTI), and urinary (UTI) tracts, as well as of surgical sites (SSI) and the blood stream (BSI), mainly in patients admitted to intensive care units (ICU), according to data collected by the National Nosocomial Infections Surveillance System (NNISS), in the United States [1,2]. The prevalence of such pathogens in nosocomial infections has varied over time; some of the changes in this pattern are attributable to health care changes, the use of antimicrobial agents, new practices of infection control, and the onset of antimicrobial resistance [3].

There is a similar pattern of isolation of such agents worldwide. This suggests that the reservoir for most of them are patients; they probably share the same risk factors for the acquisition of bacterial infections [4]. Gram-negative bacilli are the most frequent agent causing nosocomial pneumonia and urinary tract infections.

Resistance to antibiotics is a frequent finding among patients presenting with nosocomial infections. Betalactamases are enzymes often produced by GNB to evade antibiotic activity. The first enzyme within this group was identified in 1940, in an E. coli strain [5]. To date, over 130 beta-lactamases TEM (from the name of the patient who

Received on 17 January 2006; revised 13 April 2006.

Address for correspondence: Dr. Carlos Brites. Rua João das Botas, $\mathrm{S} / \mathrm{N}, 60$. andar, Laboratório de Pesquisas em Infectologia, Canela, Salvador, Bahia, Brazil, 40-110-160. Phone- 55-71-32354901 Fax: 55-71-32472756.E-mail: crbrites@ufba.br

The Brazilian Journal of Infectious Diseases 2006;10(3):191-193. (C) 2006 by The Brazilian Journal of Infectious Diseases and Contexto Publishing. All rights reserved. provided the first strain detected as producing BL) type and over 50 SHV ("sulfhydryl variable") types were identified in the Enterobacteriaceae family, most of them being $K$. pneumoniae, E. coli, and Proteus mirabilis. In addition, other GNB are able to produce such enzymes (Acinetobacter spp., for instance). Extended spectrum beta-lactamases are enzymes capable of inactivation of most beta-lactam drugs, and they usually respond only to carbapenem drugs. Usually, the Enterobacteriaceae produce chromosomically-mediated betalactamases [6]. Cephalosporins maintained their activity in the presence of such enzymes; but, in 1985, a new member this class, initially named "SHV-2", was discovered to be capable of inactivating all beta-lactamic antibiotics; it received the name ESBL

We have no information on ESBL prevalence and risk factors in our region. Consequently, we have examined the risk factors associated with infection by ESBL strains of $K$. pneumoniae, in a tertiary hospital, in Salvador, Northeast Brazil.

\section{Material and Methods}

We reviewed all positive cultures for K. pneumoniae, isolated from blood or the urinary tract, from 2000 and 2004, from patients admitted to Hospital Espanhol, a 350 bed, private tertiary hospital, in Salvador, Brazil. The Infection Control Committee records all charts originated from patients diagnosed as having nosocomial infections. We selected all nosocomial infections with a positive culture for Klebsiella pneumoniae. The diagnosis of nosocomial infection was performed according to NNISS criteria. The detection of ESBL was initially made by automated equipment (AutoScan 4 - Deide-Boehringer), and confirmed by a double disc synergy test. 
For analysis, we defined cases as those strains classified as ESBL producers, and controls as isolates negative for ESBL production. The EPI-Info databank was exported to the statistical package SAS version 6.11 for Windows. Cases and controls were paired by date of bacterial isolate (plus/minus one month). Descriptive tables and distribution of variables were analyzed by the Chi-square test and/or the Fisher exact test. Logistical regression was performed by using the stepby-step PROC LOGISTIC procedure. The variables that were statistically significant, by Wald's Chi-square test, were used for multivariate analysis. We defined a significance level of $5 \%$. We examined the length of stay in the hospital, use of corticosteroids, diagnosis of diabetes mellitus, malignancies and neutropenia, previous use of antibiotics, and renal chronic failure, as potential risk factors for ESBL. In addition, the frequency of use of invasive devices and procedures was also compared between groups.

\section{Results}

One hundred and seven patients ( 56 cases) were included in the study, with a predominance of males $(54.2 \%)$. Mean age was $63.5 \pm 19.7$ years. Cases (65.8 years) and controls (61.5 years) had similar mean ages.

Table 1 shows the frequency of the main invasive procedures in the patients included in the study. Of note, two thirds $(65.4 \%)$ of the patients had a central venous catheter (CVC), and $59.8 \%$ used a urinary catheter. Only $15 \%$ of patients diagnosed as infected by K. pneumoniae had a history of previous use of antibiotics, but $22.4 \%$ presented with a diagnosis of malignancy, $15.9 \%$ with diabetes mellitus (DM) and $15.9 \%$ with chronic renal failure (CRF).

Table 1. Frequency of main invasive procedures among patients presenting with infections by Klebsiella pneumoniae

\begin{tabular}{lcccc}
\hline Procedure & Cases & Controls & Total & P \\
\hline Urinary catheter & 34 & 30 & 64 & NS \\
Central Venous Catheter & 35 & 35 & 70 & NS \\
Mechanical ventilation & 19 & 20 & 39 & NS \\
\hline
\end{tabular}

Based on univariate analysis, the relative risk limits (95\% confidence interval) for use of mechanical ventilation (0.482.34), CVC (0.48-2.35), parenteral nutrition support (0.03$1.03)$, urinary catheter (0.79-3.79), or gastrostomy (0.98-1.1), did not differ for cases and controls. In addition, the proportion of patients older than 65 years was similar for both groups (RR: 0.72-3.39). Also, there was no detectable difference in the risk for acquisition of ESBL-producing $K$. pneumoniae (ESBL-Kp) infection for patients with neutropenia (0.438.47), with CRF (0.92-1.28) or under chemotherapy (0.855.53). Although not significant, malignancy appears to be marginally associated with infection by ESBL bacterial diseases $(0.59-1.28, \mathrm{p}=0.07$, one-tailed Fischer exact test). However, patients with a diagnosis of AIDS $(0.89-0.99)$, DM (1.34-14.71), and with a previous history of antibiotic use (1.19-13.35) were at a higher risk of infection by such agents.

Based on multivariate analysis, previous use of antibiotics $(R R=4.49, p=0.04)$, diagnosis of malignancies $(\mathrm{RR}=4.57, \mathrm{p}=0.02)$, and $\mathrm{DM}(\mathrm{RR}=5.09, \mathrm{p}=0.02)$ remained as independent risk factors for infection by ESBL-Kp. Tables 2 and 3 summarize the frequency and RR for each variable.

Table 2. Univariate analysis of the main risk factors for infection by ESBL-producing Klebsiella penumoniae

\begin{tabular}{lcc}
\hline Risk factor & OR (95\% CI) & Pvalue \\
\hline Previous antibiotic use & $4.0(1.198-13.35)$ & 0.03 \\
Chronic renal failure & $1.70(0.97-4.88)$ & $\mathrm{NS}$ \\
Use of corticosteroids & $0.91(0.83-0.99)$ & 0.056 \\
Malignant disease & $2.17(0.85-5.16)$ & 0.1 \\
Diabetes mellitus & $4.44(1.34-14.70)$ & 0.02 \\
Age greater than 60 years & $0.568(0.72-3.39)$ & 0.3 \\
\hline
\end{tabular}

$\mathrm{OR}=$ odd ratio. $\mathrm{CI}=$ confidence interval

Table 3. Multivariate analysis for risk factors associated with infection by ESBL-producing Klebsiella pneumoniae

\begin{tabular}{lccc}
\hline Risk factor & $\begin{array}{c}\text { WALD } \\
\text { chi-square }\end{array}$ & OR & Pvalue \\
\hline Parenteral Nutritional & & 0.196 & 0.2392 \\
$\quad$ Support & & & \\
Use of Corticosteroids & 0.0006 & 4.04 & 0.04 \\
Chemotherapy & 0.0008 & 0.00 & 0.98 \\
Previous use of & 4.06 & 4.96 & 0.04 \\
$\quad$ antibiotics & & & \\
Malignant Diseases & 5.060 & 4.57 & 0.01 \\
Diabetes Mellitus & 5.22 & 5.09 & 0.02 \\
Chronic Renal failure & 1.55 & 2.30 & 0.21 \\
Age $>$ 60 years & 1.58 & 0.2 & 1.86 \\
\hline
\end{tabular}

\section{Discussion}

Based on our results, the main risks for infection by ESBL$\mathrm{Kp}$ in a tertiary hospital in Salvador are previous use of antibiotics, presence of a malignant underlying disease, and DM. Other identified risks (urinary catheter, CVC, and other invasive devices, anemia, older age) were not associated with such infections $[7,8]$. However, we identified a common risk factor for these infections (previous use of antibiotics), already described by other authors $[7,8]$. On the other hand, in a large group of patients infected with ESBL-Kp, the only independent risk factor identified was the infecting organism ( $K$. pneumoniae), indicating that the epidemiology of $K$. pneumoniae may be unique, and that horizontal spread is important in the emergence of ESBL-Klebsiella strains [9]. 
Additional findings from the SENTRY project reinforced this perception: DiPersio found similarities between genotypic and phenotypic characteristics of isolates in two New York hospitals, which strongly suggests that they probably evolved into different, but genetically related clones that have the ability to cause sustained epidemic outbreaks in US medical centers [10].

In Saudi Arabia, Panhotra et al. concluded that the main risks for ESBL producing KP were advanced age, invasive devices, and previous use of third generation cephalosporins [8]. Mendelson, in Israel, found the same pattern of risk in their cohort of residents of a long-term care facility [9], and Kang, in South Korea confirmed these findings, in a large tertiary hospital in Seoul [11]. He also suggested the use of carbapenems for treatment of bacteremia by ESBL-Kp, as an approach to reduce mortality [12].

Indiscriminate use of antibiotics has been considered one of the main risks for the selection of multidrug-resistant bacteria. Third-generation cephalosporins are one of the main groups of drugs associated with increased selective pressure to generate resistant bacteria species [11-13]. Several reports confirm this conception. Lee et al. demonstrated that reduction in the use of third generation cephalosporins decreased the acquisition of ESBL-producing KP $[14,15]$. We did not evaluate the duration of use and the type of antibiotic used by patients; but the pharmacy files confirmed that the most widely-used antimicrobial drugs in the hospital were third-generation cephalosporins.

The increasing prevalence of ESBL-Kp is a common problem, worldwide [16]. In Brazil, there is a similar epidemiological picture, and the infections caused by such strains are becoming more frequent over time. We found that infections caused by ESBL-Kp are even more prevalent than those caused by non-ESBL producing $K$. pneumoniae in Salvador, Brazil. Although the retrospective design did not allow us to properly collect detailed information on the potential risk factors associated with ESBL-Kp, the coincidences between our findings and those described by other authors suggest that the epidemiology of such infections in Bahia is similar to that described elsewhere. The higher risk for patients presenting with DM or malignant diseases may reflect the severity of underlying illness, which could lead those individuals to greater use of antibiotics, or more frequent contact with health services, increasing their chance of acquiring infection by an ESBL-Kp.

In conclusion, our findings reinforce the importance of previous exposure to antibiotics as a risk factor for acquisition of a nosocomial infection caused by resistant bacteria. Programs and policies for rational antibiotic use are urgently needed, in order to minimize this important health problem. ESBL-producing Klebsiella pneumoniae infections have a significant impact on several important clinical outcomes; efforts to control outbreaks of infection with ESBL-producing K. pneumoniae should emphasize judicious use of antibiotics as well as barrier precautions to reduce spread.

\section{References}

1. Richards M.J., Edwards J.R., Culver D.H., Gaynes R.P. Nosocomial Infections in Medical Icus in the United States: National Nosocomial Infections Surveillance System. Crit Care Med 1999;27:887-92.

2. Weinstein R.A. Epidemiology and Control of Nosocomial Infections in Adult Intensive Care Units. Am J Med 1991;91(suppl 3B):1795-845.

3. Gaynes R., Edwards J.R. Overview of Nosocomial Infections Caused by Gram-negative Bacilli. Clinical Infectious Diseases 2005;41:848-54.

4. Archibald L.R. Gram-negative, Hospital-acquired Infections: A Growing Problem. Infect Control Hosp Epidemiol 2004;25:809-11.

5. Abraham E.P., Chain E. An Enzyme form Bacteria Able to Destroy Penicillin (Letter). Nature 1940;146:837.

6. Bush K., Jacoby G.A., Medeiros .AA. A Functional Classification Scheme for B-lactamases and Its Correlation With Molecular Structure. Antimicrob Agents Chemother 1995;39:1121-33.

7. Hyle E.P., Lipworth A.D., Zaoutis T.E., et al. Risk factor for increasing multidrug resistance among extendedspectrum beta-lactamase-producing Escherichia coli and Klebsiella species. Clin Infect Dis 2005;40(9):1317-24.

8. Panhotra B.R., Saxena A.K., Al-Ghamdi A.M. Extendedspectrum Beta lactamase-producing Klebsiella pneumoniae Hospital Acquired. Risk factors and Clinical Outcome. Saudi Med J 2004; 25(12):1871-6.

9. Mendelson G., Hait V., Ben-Israel J., et al. Eur J Clin Microbiol Infect Dis 2005;24(1):17-22.

10. DiPersio J.R., Desphande L.M., Biedenbach D.J., et al. Diagn Microbiol Infect Dis 2005;51(1):1-7.

11. Kang C.I., Kim S.H., Kim D.M., et al. Risk factors for and clinical outcomes of bloodstream infections caused by extended-spectrum beta-lactamase-producing Klebsiella pneumoniae. Infect Control Hosp Epidemiol 2004;25(10):860-7.

12. Kang C.I., Kim S.H., Park W.B., et al. Bloodstream infections due to extended-spectrum beta-lactamase-producing Escherichia coli and Klebsiella pneumoniae: risk factors for mortality and treatment outcome, with special emphasis on antimicrobial therapy. Antimicrob Agents Chemother 2004; 48:4574-81.

13. Fridkin S.K., Gaynes R.P. Antimicrobial Resistance in Intensive Care Units. Clin Chest Med 1999;20:303-16.

14. Meyer K.S., Urban C., Eagen J.A., et al. Nosocomial Outbreak of Klebsiella Infection Resistant to Late-generation Cephalosporins. Ann Intern Med 1993;119:353-8.

15. Lee S.-O., Lee E.S., Park S.Y., et al. Reduced Use of Thirdgeneration Cephalosporins Decreases the Acquisition of Extended-spectrum Beta-lactamase-producing Klebsiella pneumoniae. Infect Control Hosp Epidemiol 2004; 25 :832-7.

16. Streit J.M., Jones R.N., Sader H.S., Fritsche T.R. Assessment of Pathogen Occurrences and Resistance Profiles Among Infected Patients in the Intensive Care Unit: Report from the Sentry Antimicrobial Surveillance Program. Int J Antimicrob Agents 2004;24:111-8. 